\title{
ELECTRIC POLARIZATION OF ONSAGER FLUIDS. II. BIREFRINGENCE. 2. MOLAR KERR CONSTANTS OF BINARY SOLUTIONS
}

\author{
V.V. Prezhdo ${ }^{a}$, G.V. Tarasova ${ }^{b}$, O.V. Prezildo ${ }^{c}$, S.A. Tyurin ${ }^{b}$, \\ O.N. AKULOVA ${ }^{b}$ AND T.N. KURSKAYA \\ a Institute of Chemistry, University of Opole, Oleska 48, Opole, Poland \\ b Ukrainian Scientific Center for Water Control, Kharkov, Ukraine \\ ${ }^{c}$ Department of Chemistry, University of Texas, Austin, USA
}

(Received April 6, 1994; in final form June 17, 1994)

\begin{abstract}
On the ground of dielectrometry and electric birefringence data we calculated molar herr constants of substances dissolved in organic media of diverse polarity and polarizability under the conditions of infinite dilution. Minimal errors of the calculated constants, in relation to their gascous pliase valucs, were achieved as a result of the introduction of the local field model, which evolves the concepts of reactive field and local electric induction of a polarized fluid, into the orientational theory of Kerr effect.
\end{abstract}

PACS numbers: 31.70.Dk, 78.20.Fm, 72.80.Jc

\section{Introduction}

The estimation of Kerr effect is one of the most precise and highly informative melhods of investigation of fundamental properties characteristic of molecular media. In combination with the molecular refraction and light scattering techniques it enables calculation of semi-axes of molecular polarizability ellipsoids. Precise results can be obtained from the gas-phase measurements data which are not inferred with the intermolecular interaction cffects. Unfortunately, most of organic compounds are not volatile enough to perform such measurements. For many interesting chemical and biochemical investigations of electro-optical properties they are limited to infinitely diluted binary solution. Now we wish to demonstrate that such studies can provide an exact information on the polarizability of organic molecules. 


\section{Model description of molar Kerr constant of binary solution}

In this paper we present the results of experimental and calculative determination of molar Kerr constants for the substances of diverse polarity and polarizability, dissolved in non-polar and polar media.

From the experimental data on dielectrometry and electric birefringence (EB) original expression for the computation of Kerr constants of pure molecular fluids was deduced in the previous publication [1] ( $K^{1}$ is Kerr constant of a unit volume containing $N$ molecules):

$$
K^{1}=\pi N C_{\mathrm{L}}^{0} C_{A, B}^{\mathrm{e}}\left(\Theta_{1}^{\mathrm{g}}+\Theta_{2}^{\mathrm{l}}\right) \text {. }
$$

The above expression was deduced from the Langevin-Born's model with some modifications. Reactive field of an electrically polarized fluid contributes to the potential energy of molecules, hence dipole moments of free molecules $\mu_{0}$ differ from the corresponding values in liquid state $\mu_{0}^{l}$. Consequently, the dipole term $\Theta_{2}^{1}$ differs from the one $\Theta_{2}^{\mathrm{g}}$, traditionally used in literature as characteristic of gases. Morcover, the factors $C^{0}$ and $C^{\mathrm{e}}$ which establish relationship between high- and low-frequency Maxwcll's $\left(E^{0, \mathrm{e}}\right)$ and local $\left(E_{\mathrm{e}}^{0, \mathrm{e}}\right)$ ficlds $\left(E_{\mathrm{e}}^{0, \mathrm{e}}=C^{0, \mathrm{e}} E^{0, \mathrm{e}}\right)$ appear in the first and not in the second power. The factor $C^{\mathrm{e}}$ is calculated according to the suggested earlier model of local field, developing the concepts of reactive field and local electric induction of a molecular fluid $[2,3]: C^{\mathrm{e}}=C_{A}^{\mathrm{e}}$ and $C_{B}^{\mathrm{e}}$ while the common pattern of $C^{\mathrm{e}}$ is $C_{\mathrm{L}}^{\mathrm{e}}$. The factor $C^{0}$ is defined according to Lorenz $\left(C^{0}=C_{\mathrm{L}}^{0}\right)$, since the distribution of the Lorenz internal field of an optical radiation passing through a medium during EB experiments is isotropic as it has been found in [4].

Another important electro-optical characteristic of a substance is molar Kerr constant $m K^{\mathrm{g}}$ which does not contain macroscopic quantities and is connected with the constant $K^{\mathrm{g}}$ of one molecule of a gas by the formula $m K^{\mathrm{g}}=K^{\mathrm{g}} N^{-1}=$ $3 \pi N_{\mathrm{A}}\left(\Theta_{1}^{\mathrm{g}}+\Theta_{2}^{\mathrm{g}}\right)$. Analogous expression for liquids, as regarded in numerous publications [3], is $m K^{1}=K^{1} M n^{2}\left[\left(C_{\mathrm{L}}^{0}\right)^{2}\left(C_{\mathrm{L}}^{\mathrm{e}}\right)^{2} \rho\right]^{-1}$, where $N_{\mathrm{A}}$ is Avogadro number, $M$ and $\rho$ are molecular weight and density, respectively. In our approach the corresponding equation for molar Kerr constant of liquids has the form

$$
\begin{aligned}
m K^{\prime} & =3 K^{\prime} M n^{2}\left[C_{\mathrm{L}}^{0} \rho C_{A, B}^{\mathrm{e}}\right]^{-1}=3 \pi N_{\mathrm{A}}\left(\Theta_{1}^{\mathrm{g}}+\Theta_{2}^{l}\right) \\
& =m K^{\mathrm{g}}+15 \pi N_{\mathrm{A}} \alpha t(1+0.25 \alpha t)(1-\alpha t)^{2} \Theta_{2}^{\mathrm{g}},
\end{aligned}
$$

where $\alpha$ is the average dynamic polarizability of the molecules of a liquid; $t$ is the reactive field factor.

To extend the formula (1) to binary solutions let us assume that under the experimental conditions molar Kerr constant is additive over the weight factors $X_{i} ; s K_{0}^{\prime}=X_{i} s K_{i}^{\prime} ; s K_{i}^{\prime}=m K_{i}^{!} M_{i}^{-1}=3 \pi N_{\Lambda} M_{i}^{-1}\left[\Theta_{1_{i}}^{\mathrm{g}}+\left(1+1.5 \alpha_{i} t_{i}\right)^{2} \Theta_{2_{i}}^{\mathrm{g}} \times\right.$ $\left.\left(1-\alpha_{i} t_{i}\right)^{-2}\right]$, and the following parameters of the solution are linear in $X_{2}$ $\left(X_{2}<0.02\right)$ :

$$
\begin{aligned}
& K_{0}^{\prime}=K_{1}^{\prime}\left(1+\xi X_{2}\right), \quad \rho_{0}=\rho_{1}\left(1+\beta X_{2}\right), \\
& n_{0}=n_{1}\left(1+\gamma X_{2}\right), \quad \varepsilon_{C_{0}}=\varepsilon_{C_{1}}\left(1+\delta X_{2}\right),
\end{aligned}
$$


$n, \varepsilon_{C}$ - refraction index and statistic permituivity of the medium; the indices $i, j$ denote: $i=j=0$ - solution, $i=1-$ solvent, $i=2-$ solute. Denoting $s \tilde{K}_{i}^{l}=s K_{i}^{!}\left(1-\alpha_{i} \iota_{0}\right)^{-1}, i=1,2$, we can restate the assumption of additivity as follows:

$$
3 K_{0}^{1} n_{0}^{2}\left[g_{0}\left(n_{0}^{2}+2\right) \rho_{0}^{-1}\right]=X_{2} s \tilde{K}_{2}^{1}+\left(1-X_{2}\right) s \tilde{K}_{1}^{1}
$$

where $g_{0}$ is the cavity field factor. Employing independent extrapolations (2) and differentiating Eq. (3) with respect to $X_{2}$ (when $X_{2} \rightarrow 0$ ) we aclieve the expression (Eq. (4)) which makes possible to determine molar Kerr constants $\left.m \tilde{K}_{2}^{\prime}\right|_{X_{2} \rightarrow 0} \equiv$ $\infty\left(m K_{2}^{l}\right)$ of solutes from experiments on dielectrometry and EB of infinitely diluted solutions

$$
\begin{gathered}
\infty\left(m K_{2}^{\prime}\right)=\frac{3 M_{2}\left(1-\alpha_{2} l_{1}\right) n_{1}^{2}}{\rho_{1} g_{1}\left(n_{1}^{2}+2\right)} K_{1}^{\prime}\left\{1+\xi-\beta-\left[\frac{\partial}{\partial X_{2}} \ln g_{0}\right]_{\lambda_{2}=0}\right. \\
\left.-\frac{1}{1-\alpha_{1} l_{1}}\left[\frac{\partial}{\partial K_{2}} \alpha_{1} l_{0}\right]_{X_{2}=0}+\frac{4 \gamma}{n_{1}^{2}+2}\right\}-F=\infty\left(m K_{2}^{g}\right) .
\end{gathered}
$$

For non-polar $\left(\mu_{1}=0\right)$ solvents $F=0$; if $\mu_{1} \neq 0$,

$$
F=15 \pi N_{\mathrm{A}}\left(1+1.5 \alpha_{1} \iota_{1}\right)\left(1-\alpha_{2} l_{1}\right)\left(1-4 \alpha_{1} t_{1}\right)^{-1} M_{2} M_{1}^{-1}\left(\partial \alpha_{1} t_{0} / \partial X_{2}\right)_{X_{2}=0}
$$

with accuracy of $(a l)^{2}$.

A lack of reliable data on the components of $A_{i j}^{0}$ and $\mu_{0 i}$ of the majority of polar compounds incvitably compels us to restrict our studies to the molecules with the symmetry $C_{2}, C_{2 v}$, and higher, because dipole direction in these molecules is parallel to one of the axes of the $A_{i j}^{0}$ tensor. In that case the dipole term in (1) takes the form: $\Theta_{2}=\left(\mu_{02}^{1}\right)\left(2 \alpha_{l}^{0}-\alpha_{m}^{0}-\alpha_{n}^{0}\right), l \neq m \neq n=1,2,3$; where $\alpha_{p r}^{0}$ are calculated from $\vec{\alpha}_{0}$ and $\gamma^{2}$ by application of valency schemes $\left(\gamma^{2}=2 \sum_{i, j} \alpha_{i}^{0} \alpha_{j}^{0}-\right.$ $6 \sum_{i, j} \alpha_{i}^{0} \alpha_{j}^{0}$ is optical anisotropy).

\section{Experimental test of the model}

To experimentally test the expression (1) and to compare it with the results of other EB theories of liquids and known values of $m K^{\mathrm{g}}$ we measured Kerr constants $B^{l}=n K^{1} \lambda^{-1}$ ( $\lambda$ is the wavelength of an analyzing beam of light) of a series of binary extremely diluted solutions, showing weak intermolecular interaction with components which have widely varying polarities and polarizabilities. The equipment and the procedure of compensational measurements are described in $[5]$.

The outcomes of juxtaposition of gaseous phase Kcrr constants $m K_{2}^{\mathrm{g}}$ with the calculated ones $\infty\left(m K_{2}^{1}\right)_{i}$ of substances dissolved in non-polar and polar solvents are compared in Tables I and II: $\Delta m K_{2_{i}}^{\mathrm{g}}=\left|m K_{2}^{\mathrm{g}}-\infty\left(m K_{2}^{\mathrm{g}}\right)_{i}\right|\left(m K_{2}^{\mathrm{g}}\right)^{-1}$.

Therewith, according to (4), $\infty\left(m K_{2}^{\mathrm{g}}\right)_{i}=\infty\left(m K_{2}^{!}\right)_{i}+F_{i}$ and the index $i$ denotes the explicit form of the local ficld either employed within the limits of Langevin-Born's theory: $i=1$ - the Lorenz model, $i=2$ - Onsager's one, $i=3$ - Block-Walker's [6]; or used in the expression (4): $i=4$ [2], $i=5$ [3].

The data presented in 'Tables 1 and 11 demonstrate the following regularities: in accordance with the conclusions of the orientational theory and Eq. (1) 
TABLE I

Molar Kerr constants in gas pliase $\left(m K^{g}\right)$ and solvent effect $\left(\Delta m K_{2_{i}}^{g}\right)$ in non-polar media.

\begin{tabular}{|c|c|c|c|c|c|c|c|}
\hline$N$ & Substance & $\begin{array}{c}m K^{\cdot g} \\
{\left[10^{12} \mathrm{CGSE}\right]}\end{array}$ & $\Delta m K_{2_{1}}^{\mathrm{g}}$ & $\Delta m K_{22}^{\mathrm{g}}$ & $\Delta m K_{23}^{g}$ & $\Delta m K_{2_{4}}^{* g}$ & $\Delta m K_{2 \mathrm{~s}}^{\mathrm{g}}$ \\
\hline 1 & 1,4-dichlorbenzene & 36.74 & 0.614 & 0.457 & 0.206 & 0.126 & 0.098 \\
\hline 2 & $p$-xylcne & 31.10 & 0.559 & 0.429 & 0.194 & 0.093 & 0.084 \\
\hline 3 & mesitylene & 30.19 & 0.546 & 0.404 & 0.187 & 0.083 & 0.073 \\
\hline 4 & benzene & 17.16 & 0.531 & 0.392 & 0.167 & 0.076 & 0.061 \\
\hline 5 & $n$-octane & 9.64 & 0.532 & 0.387 & 0.155 & 0.071 & 0.061 \\
\hline 6 & $n$-heptane & 6.51 & 0.528 & 0.383 & 0.149 & 0.070 & 0.058 \\
\hline 7 & nitrobenzene & 1332.0 & 1.909 & 0.998 & 0.672 & 0.364 & 0.253 \\
\hline 8 & nitrometane* & 53.62 & 1.857 & 0.754 & 0.551 & 0.319 & 0.188 \\
\hline 9 & chlorobenzene ${ }^{*}$ & 191.2 & 1.772 & 0.613 & 0.468 & 0.267 & 0.163 \\
\hline 10 & o-xylene* & 47.0 & 1.593 & 0.487 & 0.372 & 0.225 & 0.119 \\
\hline 11 & toluene & 39.8 & 1.456 & 0.482 & 0.349 & 0.183 & 0.098 \\
\hline 12 & $m$-xylene & 33.5 & 1.434 & 0.474 & 0.223 & 0.179 & 0.087 \\
\hline
\end{tabular}

Solvents: cyclohexane, ${ }^{*}$ tetrachloromethane, ${ }^{* *}$ benzene.

TABLE II

Molar Kerr constants of polar compounds dissolved in non-polar media $\left(m K^{\prime 1}\right)$ and solvent effect $\left(\Delta m K_{2_{i}}\right)$ in polar media (water).

\begin{tabular}{c|l|c|c|c|c|c|c}
\hline \hline$N$ & \multicolumn{1}{|c|}{ Substance } & $\begin{array}{c}\sigma^{m} K_{2}^{+1} \\
{\left[10^{12} \mathrm{CGSE}\right]}\end{array}$ & $\Delta m K_{2_{1}}$ & $\Delta m K_{2_{2}}$ & $\Delta m K_{2_{3}}$ & $\Delta m K_{2_{4}}$ & $\Delta m K_{2_{5}}$ \\
\hline 1 & dimethylsulphoxide & 143.4 & 2.249 & 1.286 & 0.945 & 0.320 & 0.304 \\
2 & formaldoxim & $290.9^{*}$ & 2.241 & 1.294 & 0.939 & 0.317 & 0.281 \\
3 & nitromethane & $89.0^{* *}$ & 2.037 & 1.260 & 0.988 & 0.323 & 0.272 \\
4 & acetoamide & 261.3 & 2.185 & 1.237 & 0.947 & 0.298 & 0.226 \\
5 & aceton & 102.0 & 2.206 & 1.191 & 0.894 & 0.286 & 0.213 \\
6 & pyridine & $142.6^{*}$ & 2.091 & 1.174 & 0.873 & 0.286 & 0.209 \\
\hline
\end{tabular}

Non-polar solvents: tetrachloromethan, ${ }^{*}$ dioxan, ${ }^{* *}$ benzen.

the magnitudes of electro-optical constants of both pure and dissolved non-polar compounds are proportional to the optical anisotropies of molecules. Thus, for instance, the largest $m K_{2}$ pertains to $\mathrm{C}_{6} \mathrm{II}_{1} \mathrm{Cl}_{2}\left(\gamma^{2}=106 \AA^{6}\right)$ for all studied non-polar solutions, while the smallest one - to $n$-heptane $\left(\gamma^{2}=5.13 \AA^{6}\right)$. Polar substances (numbers $7 \ldots 12$ in Table I) manifest complex dependence of $m K_{2}$ on molecular properties. Nevertheless, they confirm constant's additivity with respect to anisotropic $\left(\Theta_{1}>0\right)$ and dipole $\left(\Theta>0\right.$ or $\left.\Theta_{2}<0\right)$ terms. As there exists the general tendency toward the increase in electro-optical eflect with the growth of $\mu_{0}$ and $\gamma^{2}$ of molecules of a dissolved compound: molar Kerr constant $\left(m K_{2}\right)$ of nitrobcnzene $\left(\mu_{0}=4.17 \mathrm{D}, \gamma^{2}=75 \AA^{6}\right)$ exceds that $m K_{2}$ of methylbenzene $\left(\mu_{0}=0.37 \mathrm{D}, \gamma^{2}=37 \AA^{6}\right)$. The data in Table II confim this conclusion as well. 


\section{Discussion}

The technique of infinite dilution enables us to minimize the impact of intermolecular interactions betwcen solute molecules on their properties. IIowever, solvent effect remains quite noticcable. Thus, in our experiments the influence of intermolecular (dispersive) reciprocity on the computed values of $m K_{2}$ of non-polar substances dissolved in $\mathrm{C}_{6} \mathrm{II}_{12}$ (numbers $2 \ldots 6$ in Table $\mathrm{I}$ ) reveals itself in the linear correlation between the relative errors $\Delta m K_{2}^{\mathrm{g}}$ and polarizational characteristics of molecules: $\alpha$ and $\gamma^{2}$ of $\mathrm{C}_{8} \mathrm{II}_{10}$ equal $16.6 \AA^{3}$ and $78.6 \AA^{6}$, and those of $n$-heptane $1.63 \AA^{3}$ and $5.12 \AA^{6}$, respectivcly. For the solutions of polar compounds in tetrachloromethane (numbers 8...11), which exhibit coupling of dipole-induced dipole nature, the errors $\Delta m K_{2_{i}}^{\mathrm{g}}$ augment with the enlargement of molecular moments: $\mu_{0_{2}}$ of $\mathrm{CII}_{3} \mathrm{NO}_{2}$ and $\mathrm{C}_{8} \mathrm{II}_{10}$ anount to $3.5 \mathrm{D}$ and $0.47 \mathrm{D}$, respectively. The same tendency is detected for polar solvents $\left(\mu_{0_{1}}, \mu_{0_{2}} \neq 0\right.$, Table II) with conspicuous dipole-dipole reciprocity. As it has already been intimated, the assortment of polar compounds with known $m K^{\circ}$ is sharply limitcd. This is the reason which forces us to comparison of the calculated valucs $\omega_{\infty}\left(m \mathrm{~K}_{2}^{\mathrm{g}}\right)_{i}$ of substances dissolved in water (Table II) with relevant charact.cristics $\infty_{\infty}\left(m K_{2}^{1}\right)_{i}$ of solutions with non-polar solvents. The latter have been determined by the classical orientational theory with the Lorenz local field. It is seen from Table II that the magnitudes of relative errors $\Delta m K_{2_{i}}^{\mathrm{g}}$ for polar solutcs in polar solvents are systcmatically greater than the errors for substances dissolved in non-polar solvents ('Table I), cf. for example, the solutions of nitromethane. An obvious explanation of this fact is dissimilarity of intermolecular intcractions solute-solvent.

The discrepancy between the gaseous phase values of molar EB constants and those computed from the information on binary solutions within the limits of Langevin-Born's thicory of licrr eftect is mainly conditioned by the types of model descriptions of both interrelations between Maxwell's and local fields and anisotropy of molecular polarizabilities. Introduction of Onsager's internal field, which implicilly (via the reactive field) accounts for the intermolecular coupling solute-solvent, instead of the Lorenz one into the theory of birefringence of condensed media somewhat corrects the figures $\left(m K_{2}\right)$ for all binary solutions examined: $\Delta m K_{2_{1}}^{\mathrm{g}}>\Delta m K_{2_{2}}^{\mathrm{g}}$. Subsequent evolution of this idea necds the explicit representation of the diclectric permittivity function at the boundary Onsager cavity-continuum [5]: $\Delta m K_{2_{2}}^{g}>\Delta m K_{2_{3}}^{\mathrm{g}}$. Considering inductive-orientational contributions of reactive field to the potential cnergies of molecules in a solution under the conditions of EB experiments, we obtain: $\Delta m K_{2_{3}}^{g}>\Delta m K_{2_{4}}^{g} \geq \Delta m K_{2_{5}}^{g}$, because local distribution and dielectric induction cnhances dipole moments in condensed phase. According, for instance, to the data in Table I, for the model $i=5$ relative errors in molar constants of polar substances dissolved in non-polar media are associated with the corresponding values of $m K_{2}^{\mathrm{g}}$ by the expression: ${ }_{\infty}\left(m K_{2}^{\mathrm{g}}\right)_{5} \approx\left(1+\overline{\Delta m K_{25}^{\mathrm{g}}}\right) m K_{2}^{\mathrm{g}} \approx(1+0.151) m K_{2}^{\mathrm{g}}$, while the same correlation in the case of the Lorenz local field $(i=2)$ has the form $\infty_{\infty}\left(m K_{2}^{\circ}\right)_{2} \approx(1 \pm 0.742) m K_{2}^{g}$, with the inaccuracy five times Jarger.

The data presented above indicate that the theory of EB must be completed with the theory of local ficlds which can account for the local electric induction. 
The outcomes of known approaches to the calculations of molar Kerr constants $\left(m K_{2}\right)$ of a great number of binary solutions display the following tendency:

$$
\Delta m K_{2_{1}}^{\mathrm{g}}>\Delta m K_{2_{2}}^{\mathrm{g}}>\Delta m K_{2_{3}}^{\mathrm{g}} \text {. }
$$

Our results confirm this sequence and supplement it enlancing previous developments

$$
\Delta m K_{2_{3}}^{\mathrm{g}}>\Delta m K_{2_{4}}^{\mathrm{g}} \geq \Delta m K_{2_{5}}^{\mathrm{g}} .
$$

\section{References}

[1] V.V. Prezhdo, G.V. Tarasova, O.V. Prezhdo, S.A. Tyurin, O.N. Akulova, T.N. Kurskaya, Acla Phys. Pol. A 85, 794 (1994).

[2] S.A. Tyurin, M.V. IIastchina, Yu.D. Feldman, Acla Phys. Pol. A 70, 697 (1986).

[3] S.A. Tyurin, M.V. IIastchina, Zh. Fiz. K'him. (USSR) 61, 114 (1987).

[4] M.F. Vuks, N.B. Rozhdestvenskaya, K. Eyducr, Opt. Speklrosk. (USSR) 45, 114 (1978).

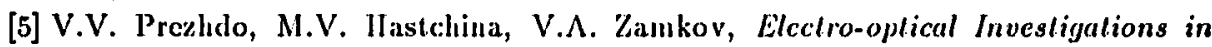
Physics and Chemistry, Vyssha Shkola (KSU), Kharkov 1982, p. 152 (in Russian).

[6] II. Block, Z.M. Walker, Chem. Phys. Lell. 19, 363 (1973). 